\title{
Capacity Scaling of Wireless Social Networks
}

\author{
Cheng Wang, Lu Shao, Zhong Li, Lei Yang, Xiang-Yang Li, Senior Member, IEEE, and Changjun Jiang
}

\begin{abstract}
In this paper, we investigate capacity scaling laws of wireless social networks under the social-based session formation. We model a wireless social network as a three-layered structure, consisting of the physical layer, social layer, and session layer; and we introduce a cross-layer distance\&density-aware model, called the population-based formation model, under which: 1) for each node $v_{k}$, the number of its friends/followers, denoted by $q_{k}$, follows a Zipf's distribution with degree clustering exponent $\gamma$; 2) $q_{k}$ anchor points are independently chosen according to a probability distribution with density function proportional to $\left(\mathbf{E}_{k, X}\right)^{-\beta}$, where $\mathbf{E}_{k, X}$ is the expected number of nodes (population) within the distance $\left|v_{k}-X\right|$ to $v_{k}$, and $\beta$ is the clustering exponent of friendship formation; 3) finally, $q_{k}$ nodes respectively nearest to those $q_{k}$ anchor points are selected as the friends of $v_{k}$. We present the general density function of social relationship distribution, with general distribution of physical layer, serving as the basis for studying general capacity of wireless social networks. As the first step of addressing this issue, for the homogeneous physical layer, we derive the social-broadcast capacity under both generalized physical and protocol interference models, taking into account general clustering exponents of both friendship degree and friendship formation in a 2-dimensional parameter space, i.e., $(\gamma, \beta) \in[0, \infty)^{2}$. Importantly, we notice that the adopted model with homogenous physical layer does not sufficiently reflect the advantages of the population-based formation model in terms of realistic validity and practicability. Accordingly, we introduce a random network model, called the center-clustering random model (CCRM) with node distribution exponent $\delta \in[0, \infty)$, highlighting the clustering and inhomogeneity property in real-life networks, and discuss how to further derive more general network capacity over 3-dimensional parameter space $(\delta, \gamma, \beta) \in[0, \infty)^{3}$ based on our results over $(\gamma, \beta) \in[0, \infty)^{2}$.
\end{abstract}

Index Terms-Scaling Laws, Social Networks, Wireless Networks, Network Capacity.

\section{INTRODUCTION}

Wireless networks are generally the wireless communication implementations for real-life networking applications. Then, research issues of wireless networks usually come from and aim at the challenges of wireless technology in specific applications, e.g., wireless sensor networks, wireless local area networks, and wireless social networks, a wireless implementation of social networks, which is the focus of this work. In social networks, the relationship/edge between users/vertices represents a specific interdependency, such as co-authorship, citationship, or friendship, and so on. Based on massive datasets of large-scale real-world online social networks, such as Myspace [1], Twitter [2], Flickr [3], LiveJournal [4], and Facebook [5], extensive studies validate respectively that these two most representative features of complex networks, i.e., the small-world phenomenon and scale-free degree distribution, nearly hold in online social networks. Wireless social networks can be analyzed from a layered perspective, i.e., the social network of users can be regarded as an overlay network over their physical communication network. Therefore, it is necessary for studying wireless social networks to take the property of social networks into account.

- Wang, Shao, Zhong Li, and Jiang are with the Department of Computer Science and Technology, Tongji University, and with the Key Laboratory of Embedded System and Service Computing, Ministry of Education,China.(E-mail: 3chengwang@gmail.com,shaolu1991@gmail.com, lizhong007@hotmail.com,cjjiang@tongji.edu.cn)

- Yang is with the Institute of Trustworthy Networks and Systems, Tsinghua University. (E-mail: young@tagsys.org)

- Xiang-Yang Li is with the Department of Computer Science and Technology and TNLIST, Tsinghua University, and is also with the Department of Computer Science, Illinois Institute of Technology. (E-mail: xli@cs.iit.edu)
As online social networking services are becoming more and more popular and the adoption rates of smart wireless devices like smartphones are increasing aggressively, wireless social network applications will be recognized as the typical instances of large-scale wireless networks. Therefore, it is significant to investigate the fundamental limits of such a large-scale wireless system. As an important metric of fundamental limits, capacity scaling laws of wireless networks, i.e., the scaling of the throughput capacity in the limit when the size of network gets large, have been extensively studied in depth from both theoretical and practical perspectives, [6][12], since Gupta and Kumar [13] took the lead to study the unicast capacity for homogeneous wireless random and arbitrary networks. In wireless social networks, source-destination associations depend on their social relationships that had been shown to be inhomogeneous, however, traffic sessions are traditionally assumed to be formed independently in a uniformly random fashion in the literature, which makes the existing results not applicable to wireless social networks. Accordingly, this paper aims to introduce the social-based session formation model conforming with wireless social networks, and derive the corresponding capacity scaling laws.

Main Challenges and Our Solutions: We list the following three main challenges in addressing the problem.

Challenge I: Modeling Social Formation Based on Geography. On the one hand, since the distance over which data are carried under every session is one of key factors determining the throughput capacity of network, the session formation model should be necessarily relevant to the geography. On the other hand, some classical experiments showed or implied that social relationships among users strongly depend on their geographic location, [14]-[16]. Thus, under the assumption that a 


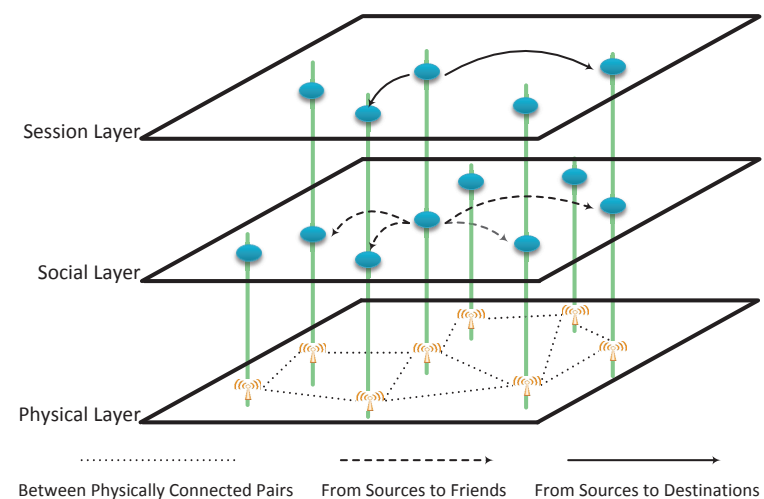

Fig. 1. Layered System Model.

session is built only among the users with social relationships (We also call them "friends" here.), i.e., the source chooses its destination only from its friends, a geography-based social relationship formation model acts as the precondition for the session formation model. Some theoretical models have been advanced to model the social relationship formation depending on geography in social networks. Kleinberg [17] initiated a distance-based social model relating geographical distance and social friendship, in which the probability of befriending a particular user is inversely proportional to the positive power of the distance. In [18] and [19], the distance-based social model had been adopted in studying the unicast capacity of wireless social networks. Intuitively, such a session pattern leads to a mismatch with the characteristics of data traffic in real life social networks. Furthermore, recent experimental studies showed that the distance-based models do not really accord with the distribution of social relationships in social networks, [20]. Therefor, it is necessary to derive the more practical results on capacity scaling of wireless social networks by introducing more realistic social models.

Liben-Nowell et al. [20] stated that the social formation indeed depends on both distance and density, and introduced the rank-based model, where the probability of befriending a particular person is inversely proportional to the positive power of the number of closer people. To the best of our knowledge, the rank-based model is the most realistic theoretical model for social formation. But it still has shortcoming on analyzing capacity scaling laws in terms of the convenience of analysis or theoretical basis and rigor, because it directly selects nodes instead of points/positions, which leads that the distances of sessions are dependent and then causes analysis difficulty in bounding the sum of transport distances of sessions with multiple destinations, [13], [21]. Then, for addressing the capacity scaling laws of wireless networks, it should be the first step to introduce a new distance\&density-aware social formation model that is suitable for capacity analysis, while keeping the advantages of rank-based model.

Our Solution for Challenge I: We model a wireless social network as a three-layered structure, consisting of the physical layer, social layer, and session layer, as illustrated in Fig.1. On the basis of rank-based model, we present a cross-layer distance\&density-aware social model good at the analysis of capacity scaling laws, called the population- based formation model $\mathbb{P}(\delta, \gamma, \beta)$, where $\delta, \gamma$, and $\beta$ are the clustering exponents of node distribution, friendship degree and friendship formation, respectively. Under $\mathbb{P}(\delta, \gamma, \beta): 1)$ for each node $v_{k}$, the number of its friends, denoted by $q_{k}$, follows a Zipf's distribution with friendship degree clustering exponent $\gamma$;2) $q_{k}$ anchor points are independently chosen according to a probability distribution with density function proportional to $\mathbf{E}_{k, X}^{-\beta}$, where $\mathbf{E}_{k, X}$ is the expected number of nodes (population) within the distance $\left|v_{k}-X\right|$ to $v_{k}$, and $\beta$ is the clustering exponent of friendship formation; 3) finally, select $q_{k}$ nodes respectively nearest to those $q_{k}$ anchor points as the friends of $v_{k}$. Please refer to Section 2.2.2 for the detailed discussion on the advantages of this model.

Challenge II: Bounding Sum of Distances for All Sessions. The sum of transport distances for all sessions is the prerequisite for bounding the capacity scaling laws, [13], [22]. The long-tailed property of both the destination number (Zipf's Distribution) and destination distribution (Power Law Distribution) causes the transport distances of resulted sessions to be significantly inhomogeneous, which makes it more difficult to bound such a sum.

Our Solution for Challenge II: We present the density function of general social friendships distribution (Theorem 1) and bounds on length of Euclidean spanning trees over nodes chosen according to this density function (Theorem 2). The results can act as the basis for addressing the capacity of wireless social networks.

Challenge III: Deriving General Results on Capacity Scaling Laws. The complete result under the system model includes the capacity for every point in the 3-dimensional parameter space, i.e., $(\delta, \gamma, \beta) \in[0, \infty)^{3}$. The complexity of analysis is substantially increased due to the involvement of multiple clustering exponents.

Our Solution for Challenge III: As the first work under this general model, this paper derives the capacity for socialbroadcast sessions under the model $\mathbb{P}(\delta=0, \gamma, \beta)$, taking into account general clustering exponents of both friendship degree and friendship formation, i.e., $(\gamma, \beta) \in[0, \infty)^{2}$ (Theorem 3 ). In addition, we probe the feasibility of studying network capacity under the social model with inhomogeneous physical layer, by extending the basic theorem (Theorem 1) for the general distribution of anchor points.

The rest of the paper is organized as follows. In Section 2, we introduce the network model. In Section 3, we present the preliminary results for the anchor points distributed in the center-clustering random model (CCRM) according to the proposed population-based formation scheme, which lay the foundation for addressing capacity scaling laws for populationbased social formation model on general physical layers. Based on these results, we derive the social-broadcast capacity for homogeneous physical layer in Section 4, acting as the first step of investigating general results on the capacity of wireless social networks. Finally, we conclude the paper and discuss some topics for future research in Section 5.

\section{System Model}

We present a three-layer perspective for the wireless social network with social-based sessions, consisting of the physical 
layer, social layer and session layer, as in Fig.1.

Throughout the paper, we let $\mathbf{E}[X]$ denote the mean of a random variable $X$.

\subsection{Physical Layer Deployment}

We introduce a random network model, called the centerclustering random model (CCRM), highlighting the clustering and inhomogeneity property in real-life networks.

\subsubsection{Center-Clustering Random Model (CCRM)}

We consider the network composed of a random number of $N$ wireless ad hoc nodes/users distributed over a square region of area $S:=n$, where $\mathbf{E}[N]=n$. To avoid border effects, we consider wraparound conditions at the network edges, i.e., the network area is assumed to be the surface of a two-dimensional Torus $\mathcal{O}$. To simplify the description, we assume that the number of nodes is exactly $n$, and denote the set of nodes by $\mathcal{V}=\left\{v_{k}\right\}_{k=1}^{n}$, without changing our results in order sense, [6], [7], [11].

To emulate the clustering behavior of users distribution in wireless networks, we construct the center-clustering random model (CCRM) by the following procedure: First, making a center of $\mathcal{O}$ as the center point, denoted by $O$. Then, the center point $O$ generates a point process of nodes whose local intensity at position $X$ is given by $\mathbf{d}(X)=n \cdot \kappa(O, X)$, where $\kappa(O, \cdot)$ is a dispersion density function. As in the literature, we restrict ourselves to the kernel $\kappa(O, \cdot)$ that is invariant under both translation and rotation, i.e., $\kappa(O, X)=\kappa(|X-O|)$ depends only on the Euclidean distance $|X-O|$ of point $X$ from the cluster center $O$, [23], [24]. Moreover, we assume that $\kappa(O, \cdot)$ is a summable, non-increasing, bounded and continuous function, and $\int_{\mathcal{O}} \kappa(O, X) d X=1$. Following a common normalizing method, the kernel can be specified by first defining a non-increasing, bounded and continuous function $g(s)$ and then normalizing it over the area $\mathcal{O}$ :

$$
\kappa(O, X)=\frac{g(|X-O|)}{\int_{\mathcal{O}} g(|Y-O|) d Y} .
$$

Then, we can conclude that a center-clustering random model (CCRM) is determined by three factors/parameters, i.e., the number of all nodes $n$, the area of deployment region $S$, and the critical parameter of dispersion density function $g(\cdot)$. We denote a CCRM by $\mathcal{N}(n, S ; g(\cdot))$ in this paper.

Specifically, we define $g(s):=\min \left\{1, s^{-\delta}\right\}$, where $\delta \in$ $[0, \infty)$ is the clustering exponent of node distribution. Note that when $\delta=0$, the model degenerates into the homogeneous random extended network, [6], [25].

We introduce an approximate real-world case of CCRM based on Google+ dataset. Please see details in Appendix D.2.

\subsubsection{Extending Generality of Physical Layer Model}

In this paper, we only study the capacity scaling laws under the CCRM with extended scaling pattern, where there is only one center point. We notice that based on the CCRM, one can develop more general physical layer models in terms of clustering patterns and scaling patterns. To smoothen the mainline of our work, we have moved the details of extended discussion to Section 5.2.

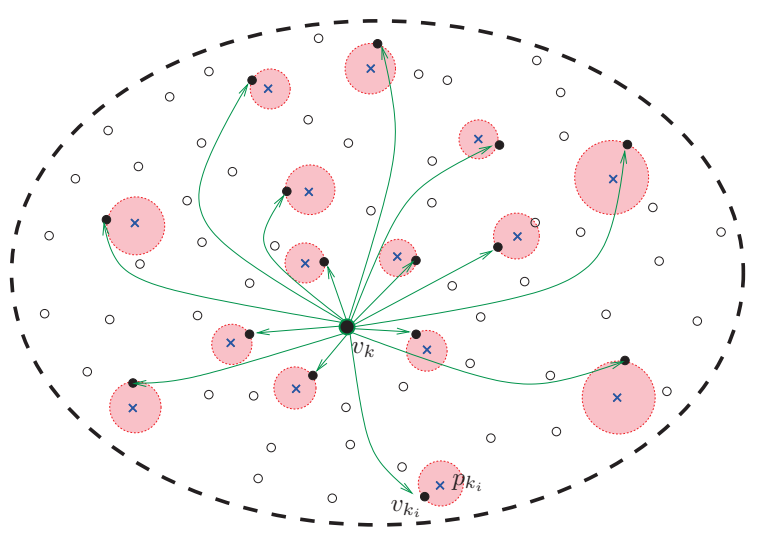

Fig. 2. Friendships of Source $v_{k}$. Making the position of node $v_{k}$ as the reference point, choose independently $q_{k}$ anchor points on the torus region $\mathcal{O}$, denoted by $p_{k_{i}}$ with $1 \leq i \leq q_{k}$, according to a probability density function as in Eq.(2). The $q_{k}$ friends of $v_{k}$ are located according to the positions of the corresponding anchor points.

\subsection{Social Layer Formation}

We introduce a social formation model, called populationbased social model. We will clarify the advantages of this model later in Section 2.2.2.

\subsubsection{Population-based Social Formation Model}

Let $\mathcal{D}(u, r)$ denote the disk centered at a point $u$ with radius $r$ in the deployment region $\mathcal{O}$, and let $N(u, r)$ denote the number of nodes contained in $\mathcal{D}(u, r)$.

For a node $v_{k} \in \mathcal{V}$, construct its friendship set of $q_{k}, q_{k} \geq$ 1 , nodes/friends, say $\mathcal{F}_{k}$, by the following procedure:

1. Zipf's Degree Distribution of Social Relations: Assume that the number of friends (or followers) of a particular node $v_{k} \in \mathcal{V}$, denoted by $q_{k}$, follows a Zipf's distribution [26], i.e.,

$$
\operatorname{Pr}\left(q_{k}=l\right)=\left(\sum_{j=1}^{n-1} j^{-\gamma}\right)^{-1} \cdot l^{-\gamma}
$$

From Eq.(1), we can observe that the degree distribution above depends on the specific network size (the number of users $n$ ). Please see a numerical validation based on Google+ dataset for the Zipf's degree distribution in Appendix D.3.

2. Population-Based Formation of Social Relations: Making the position of node $v_{k}$ as the reference point, choose $q_{k}$ points independently on the torus region $\mathcal{O}$ according to a probability distribution with density function:

$$
f_{v_{k}}(X)=\Phi_{k}(S, \beta) \cdot\left(\mathbf{E}\left[N\left(v_{k},\left|X-v_{k}\right|\right)\right]+1\right)^{-\beta},
$$

where the random variable $X:=(x, y)$ denotes the position of a selected point in the deployment region, $\left|X-v_{k}\right|$ denotes the Euclidean distance between point $X$ and node $v_{k}, \beta \in[0, \infty)$ represents the clustering exponent of friendship formation; the coefficient $\Phi_{k}(S, \beta)>0$ depends on $\beta$ and $S$ (the area of deployment region), satisfying that:

$$
\Phi_{k}(S, \beta) \cdot \int_{\mathcal{O}}\left(\mathbf{E}\left[N\left(v_{k},\left|X-v_{k}\right|\right)\right]+1\right)^{-\beta} d X=1 .
$$

3. Nearest-Principle Position of Friends: Let $\mathcal{A}_{k}=$ $\left\{p_{k_{i}}\right\}_{i=1}^{q_{k}}$ denote the set of these $q_{k}$ points. Let $v_{k_{i}}$ be the 


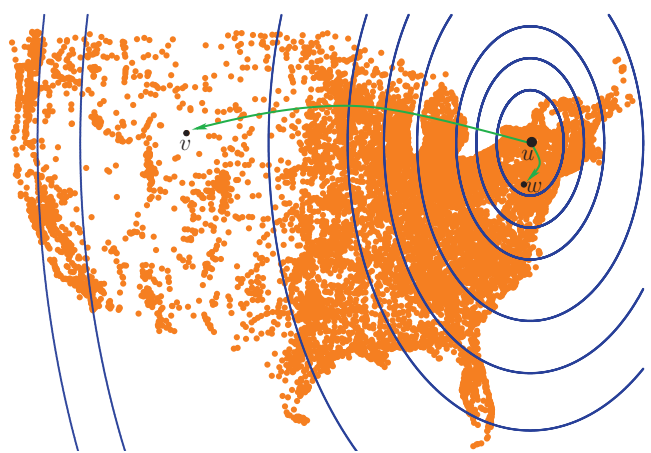

Fig. 3. Inhomogeneity of LiveJournal Population [20]. A dot is shown for every distinct United States location home to at least one LiveJournal user (up to Feb. 2004). The population of each successive displayed circle (all centered on Ithaca, NY) increases by 50,000 people. The friendships of $u \rightarrow v$ and $u \rightarrow w$ are respectively underestimated and overestimated by the distance-based model.

nearest node to $p_{k_{i}}$, for $1 \leq i \leq q_{k}$ (ties are broken randomly). Denote the set of these $q_{k}$ nodes by $\mathcal{F}_{k}=\left\{v_{k_{i}}\right\}_{i=1}^{q_{k}}$. Please see the illustration in Fig.2. We call point $p_{k_{i}}$ the anchor point of $v_{k_{i}}$, and define a set $\mathcal{P}_{k}:=\left\{v_{k}\right\} \cup \mathcal{A}_{k}$.

Throughout this paper, we use $\mathbb{P}(\delta, \gamma, \beta)$ to denote the population-based social model.

\subsubsection{Advantages of Population-Based Model}

After Kleinberg [17] proposed a distance-based social model relating geographical distance and social friendship, LibenNowell et al. [20] introduced the rank-based model, where the probability of befriending a particular person is inversely proportional to the power of the number of closer people. They validated the practicality of rank-based model by analyzing the data of an online social network, the LiveJournal online community. They pointed out that the weakness of distancebased models lies in that for a particular user, it underestimates the friendship probability of the distant nodes in the lowdensity region, when the geographical distribution of users is inhomogeneous in common occurrence, as illustrated in Fig.3. In addition, we also give a validation of rank-based model based on Google+ dataset in Appendix D.4.

The rank-based model states that the friendship probability depends on both the geographic distance and node density. Following this observation, by modifying the rank-based model, we propose the distance\&density-aware populationbased social model. We highlight that the population-based model is more convenient and systematic for addressing the issue of capacity scaling laws. Anchor points are usefully introduced, in order to ensure the independence of length of certain Euclidean spanning trees, thus makes it convenient to bound the total length, e.g., the proof of Lemma 6. However, under the rank-based model where the friendships are directly built over nodes without anchor points, the corresponding independence cannot be guaranteed, which usually brings the difficulty on the theoretical rigor. The advantages of the pointbased model for the basis and rigor in analysis, compared to the node-based model, had been apparent in [13], [21].

\subsection{Session Layer Construction}

After the social layer is formed, social sessions can be defined according to the specific applications: For the socialunicast/social-multicast, the source node delivers message to one/multiple selected friend(s).

For the social-broadcast, the source node broadcasts message to all its friends, such as tweets in Twitter and posts in Facebook. Accordingly, we can define other session patterns based on the definitions of corresponding non-social sessions, such as social-anycast [27] and social-manycast [28].

In this work, we mainly study social-broadcast sessions.

\subsection{Communication Model}

We mainly adopt the generalized physical model [6], [21] due to its generality and practicality compared to other models like the protocol model and physical model [13]. Let $\mathcal{L}_{t}$ denote a scheduling set of links in which all links can be scheduled simultaneously in time slot $t$; let $\alpha>2$ denote the power attenuation exponent; let $B$ and $P$ denote the bandwidth and transmitting power, respectively.

Definition 1: Under the generalized physical model, when a scheduling set $\mathcal{L}_{t}$ is scheduled, the rate of a link $\langle u, v\rangle \in$ $\mathcal{L}_{t}$ is achieved at

$$
R_{u, v ; t}=B \times \mathbf{1} \cdot\left\{\langle u, v\rangle \in \mathcal{L}_{t}\right\} \times \log \left(1+\operatorname{SINR}_{u, v ; t}\right),
$$

where $\mathrm{SINR}_{u, v ; t}=\frac{P \cdot \ell(|u-v|)}{N_{0}+\sum_{<i, j>\epsilon \mathcal{L}_{t} /<u, v>} P \cdot \ell(|i-v|)}, N_{0}$ denotes the background noise, $|u-v|$ represents the Euclidean distance between nodes $u$ and $v ; \ell(\cdot)$ denotes the power attenuation function that is assumed to depend only on the distance between the transmitter and receiver, to be specific, $\ell(|\cdot|):=|\cdot|^{-\alpha}$ for dense networks, and $\ell(|\cdot|):=\min \left\{1,|\cdot|^{-\alpha}\right\}$ for extended networks.

\subsection{Network Capacity for Social Sessions}

Denote a session by $\mathcal{S}_{k}:=\left\{v_{k}\right\} \cup \mathcal{D}_{k}$, where $\mathcal{D}_{k} \subseteq \mathcal{F}_{k}=$ $\left\{v_{k_{i}}\right\}_{i=1}^{q_{k}}$ is the set of destinations of $v_{k}$. For social-broadcast sessions, it holds that $\mathcal{D}_{k}=\mathcal{F}_{k}$.

Let $\Lambda=\left(\lambda_{1}, \lambda_{2}, \cdots, \lambda_{n}\right)$ denote a rate vector of the data rate of all sessions. A rate vector $\Lambda$ is feasible if there is a $T<\infty$ such that in every time interval (with unit seconds) $[(t-1) \cdot T, t \cdot T]$, every source node $v_{k}$ can send $T \cdot \lambda_{k}$ bits to all its destinations. For a rate vector, we define the per-session throughput as $\Lambda(n)=\min _{v_{k} \in \mathcal{V}} \lambda_{k}$.

Definition 2 (Achievable Throughput): We say a persession throughput $\Lambda(n)$ is achievable for all social sessions if there is a feasible rate vector $\Lambda=\left(\lambda_{1}, \lambda_{2}, \cdots, \lambda_{n}\right)$ such that $\Lambda(n)=\min _{v_{k} \in \mathcal{V}} \lambda_{k}$.

Definition 3 (Social Capacity): The per-session capacity for a class of random networks is of order $\Theta(\Gamma(n))$ if there are constants $0<\underline{c}<\bar{c}<+\infty$ such that

$$
\begin{aligned}
& \lim _{n \rightarrow+\infty} \operatorname{Pr}(\Lambda(n)=\underline{c} \cdot \Gamma(n) \text { is achievable })=1, \\
& \liminf _{n \rightarrow+\infty} \operatorname{Pr}(\Lambda(n)=\bar{c} \cdot \Gamma(n) \text { is achievable })<1 .
\end{aligned}
$$

To facilitate the reader, we have reported in Table 1 a collection of frequently-used system parameters. 
TABLE 1

Notations for Exponents

\begin{tabular}{l|l}
\hline Notation & Definition \\
\hline \hline$\delta \in[0, \infty)$ & clustering exponent of node distribution \\
\hline$\gamma \in[0, \infty)$ & clustering exponent of friendship degree \\
\hline$\beta \in[0, \infty)$ & clustering exponent of friendship formation \\
\hline$\alpha \in(2, \infty)$ & attenuation exponent of signal transmission \\
\hline
\end{tabular}

\section{Preliminary Results for Distribution OF ANCHOR POINTS}

In this section, we mainly present some preliminary results for the anchor points distributed according to the population-based social formation scheme in the center-clustering random model (CCRM). The results can serve as the basis for addressing the capacity of wireless social networks under the general CCRM and population-based social formation model.

In the center-clustering random model (CCRM), say $\mathcal{N}(n, S ; g(\cdot))$, we construct a set of $q+1$ anchor points, denoted by $\mathcal{P}=\left\{X_{i}\right\}_{i=0}^{q}$, by the following procedure: 1 . Select arbitrarily a point from $\mathcal{O}$ as the first one in $\mathcal{P}$, denoted by $X_{0} .2$. Making point $X_{0}$ as the reference point denoted by $O^{\prime}$, select independently other $q$ points at random according to the probability distribution with density function as described in Eq.(2) of the population-based formation model (Let $v_{k}:=X_{0}$ ).

Let $\mathcal{A}:=\left\{X_{i}\right\}_{i=1}^{q}$. Next, we propose two theorems, and prove them in Section 3.3.

\subsection{General Distribution Density Function}

For the probability density function of point distribution, we have Theorem 1.

Theorem 1: Making the point $X_{0}$ as the reference point $O^{\prime}$, the distribution of points in $\mathcal{A}=\left\{X_{i}\right\}_{i=1}^{q}$ follows the probability with density function

$$
f_{X_{0}}(X)=\frac{\left[\int_{\mathcal{D}\left(X_{0},\left|X-X_{0}\right|\right)} \mathbf{d}(Y) d Y+1\right]^{-\beta}}{\int_{\mathcal{O}}\left[\int_{\mathcal{D}\left(X_{0},\left|Z-X_{0}\right|\right)} \mathbf{d}(Y) d Y+1\right]^{-\beta} d Z}
$$

where $\mathbf{d}(Y)=n \cdot \frac{\min \left\{1,|Y-O|^{-\delta}\right\}}{\int_{\mathcal{O}} \min \left\{1,|Z-O|^{-\delta}\right\} d Z}$.

\subsection{Euclidean Minimum Spanning Tree}

For the Euclidean minimum spanning trees of $\mathcal{P}$ and $\mathcal{A}$, denoted by $\operatorname{EMST}(\mathcal{P})$ and $\operatorname{EMST}(\mathcal{A})$, respectively, we have Theorem 2.

Theorem 2: As $q \rightarrow \infty$ : With probability 1 , it holds that

$$
|\operatorname{EMST}(\mathcal{A})|=\Theta\left(\sqrt{q} \cdot \int_{\mathcal{O}} \sqrt{f_{X_{0}}(X)} d X\right) ;
$$

with high probability $1-o(1 / \hat{N})$, it holds that

$$
|\operatorname{EMST}(\mathcal{P})|:[|\operatorname{EMST}(\mathcal{A})|,|\operatorname{EMST}(\mathcal{A})|+\bar{L}],{ }^{1}
$$

1. We use the term $f(n):[\underline{\phi}(n), \bar{\phi}(n)]$ to represent $f(n)=\Omega(\underline{\phi}(n))$ and $f(n)=O(\bar{\phi}(n))$; and use $f(n):(\underline{\phi}(n), \bar{\phi}(n))$ to represent $f(n)=$ $\omega(\phi(n))$ and $f(n)=o(\bar{\phi}(n))$. where $f_{X_{0}}(X)$ is defined in Theorem 1 , and

$$
\bar{L}=\min \left\{L \mid \int_{\mathcal{D}\left(X_{0}, L\right)} f_{X_{0}}(X) d X=\Omega\left(\min \left\{\frac{\log \hat{N}}{q}, 1\right\}\right)\right\}
$$

with $\hat{N}:(1, n]$ is a given parameter.

Note that the parameter $\hat{N}$ can be defined as the number of nodes with degree of order $\omega(1)$.

\subsection{Proof of Theorem 1 and Theorem 2}

First of all, the cost of an edge $\left(X_{i}, X_{j}\right)$ is given by $\Psi\left(\left|X_{i}-X_{j}\right|\right)=\left|X_{i}-X_{j}\right|$, that is, the exponent $\sigma$ in Lemma A.1 equals 1 . In addition, $\Psi(x)$ is a monotonically increasing function. Let $L$ denote the distance between the center $O$ and reference point. Then, under the center-clustering random model $\mathcal{N}(n, S ; g(\cdot))$, by Eqs.(2) and (3), the density function is specified into Eq.(4). Then, by Lemma A.1, we get that

$$
|\operatorname{EMST}(\mathcal{A})|=\Theta\left(\sqrt{q} \cdot \int_{\mathcal{O}} \sqrt{f_{X_{0}}(X)} d X\right) .
$$

It is straightforward that $|\operatorname{EMST}(\mathcal{P})|=\Omega(|\operatorname{EMST}(\mathcal{A})|)$. On the other hand, let $\underline{L}$ denote the smallest distance from the points in $\mathcal{A}$ to point $X_{0}$. Then,

$$
\left(1-\int_{\mathcal{D}\left(X_{0}, \underline{L}\right)} f_{X_{0}}(X) d X\right)^{q}=o(1) .
$$

That is, $\int_{\mathcal{D}\left(X_{0}, \underline{L}\right)} f_{X_{0}}(X) d X=\omega(1 / q)$. Thus, $\underline{L} \leq \bar{L}$, where $\bar{L}$ is defined in Eq.(7), which completes the proof. Note that we deliberately relax the upper bound of $\underline{L}$ as in Eq.(7) in order to ensure Eq.(6) to hold with uniformly high probability for $\Theta(n)$ Euclidean spanning trees, [29].

\section{Social-Broadcast CAPACITY FOR Ho- MOGENEOUS PHYSICAL LAYER}

In this work, we specifically reduce the complexity from three dimensions $(\delta, \gamma, \beta) \in[0, \infty)^{3}$ to two dimensions $(\gamma, \beta) \in$ $[0, \infty)^{2}$ by letting $\delta=0$. In this case of extremely weak clustering behavior, the physical layer degenerates into the homogeneous random network model, [6], [13], [30], where $\mathbf{d}(Y) \equiv \Theta(1)$.

\subsection{Main Results}

\subsubsection{Capacity under Generalized Physical Model}

Theorem 3: Under the population-based social model $\mathbb{P}(\delta=0, \gamma, \beta)$ and the generalized physical model (GphyM) with $\alpha>2$, the per-session social-broadcast capacity is of order $\Lambda$, where $\Lambda$ is defined in Table.2.

From Theorem 3, there are still gaps between upper and lower bounds on social-broadcast capacity under the generalized physical model in four regimes. As illustrated in Fig.4, these four regimes are indeed lines in the 2-dimensional parameter space $(\gamma, \beta) \in[0, \infty)^{2}$. A challenging issue is to close those gaps by presenting possibly new tighter upper and lower bounds by using some new arguments or designing new schemes. 
TABLE 2

Social-Broadcast Capacity under GphyM

\begin{tabular}{|c|c|}
\hline$\gamma$ & Social Capacity under GphyM $-\Lambda$ : \\
\hline$\gamma>2$ & $\begin{cases}\Theta\left((\log n)^{-\frac{\alpha}{2}}\right), & \beta>2 ; \\
{\left[(\log n)^{-\frac{\alpha+1}{2}},(\log n)^{-\frac{\alpha}{2}}\right],} & \beta=2 ; \\
\Theta\left(1 / n^{1-\frac{\beta}{2}}\right), & 1<\beta<2 \\
\Theta(\sqrt{\log n} / \sqrt{n}), & \beta=1 ; \\
\Theta(1 / \sqrt{n}), & 0 \leq \beta<1\end{cases}$ \\
\hline$\gamma=2$ & $\begin{array}{ll}{\left[(\log n)^{-\frac{\alpha+3}{2}},(\log n)^{-\frac{\alpha}{2}-1}\right],} & \beta \geq 2 ; \\
\Theta\left(1 / n^{1-\frac{\beta}{2}}\right), & 1<\beta<2 ; \\
\Theta(\sqrt{\log n} / \sqrt{n}), & \beta=1 ; \\
\Theta(1 / \sqrt{n}), & 0 \leq \beta<1 .\end{array}$ \\
\hline $3 / 2<\gamma<2$ & $\begin{array}{ll}\Theta\left((\log n)^{-\frac{\alpha}{2}} / n^{2-\gamma}\right), & \beta \geq 2 \gamma-2 \\
\Theta\left(1 / n^{1-\frac{\beta}{2}}\right), & 1<\beta<2 \gamma-2 \\
\Theta(\sqrt{\log n} / \sqrt{n}), & \beta=1 \\
\Theta(1 / \sqrt{n}), & 0 \leq \beta<1\end{array}$ \\
\hline$\gamma=3 / 2$ & $\begin{cases}\Theta\left((\log n)^{-\frac{\alpha}{2}} / \sqrt{n}\right), & \beta \geq 1 ; \\
{\left[\frac{(\log n)^{-\frac{\alpha+1}{2}}}{\sqrt{n}}, \frac{(\log n)^{-\frac{\alpha}{2}}}{\sqrt{n}}\right],} & 0 \leq \beta<1 .\end{cases}$ \\
\hline $1<\gamma<3 / 2$ & $\Theta\left((\log n)^{-\frac{\alpha}{2}} / n^{2-\gamma}\right)$ \\
\hline$\gamma=1$ & {$\left[(\log n)^{\frac{1-\alpha}{2}} / n,(\log n)^{1-\frac{\alpha}{2}} / n\right]$} \\
\hline $0 \leq \gamma<1$ & $\Theta\left((\log n)^{-\frac{\alpha}{2}} / n\right)$ \\
\hline
\end{tabular}

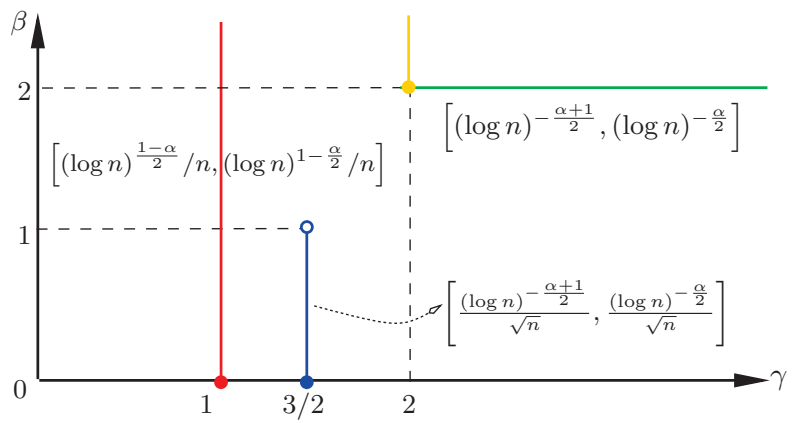

Fig. 4. Four Regimes/Lines Where Gaps Exist.

\subsubsection{Capacity under Protocol Model}

We concentrate on deriving the capacity under the generalized physical model, while for completeness, we also include the results on capacity under the well-known protocol model (ProM, [13]). Based on our results on EMSTs and ESTs of social-broadcast sessions (Lemma 6 and Lemma 9), using the analytical methods for capacity under the protocol model in [13], [22], we get that

Theorem 4: Under the population-based social model $\mathbb{P}(\delta=0, \gamma, \beta)$ and the ProM, the per-session social-broadcast capacity is of order $\Lambda^{\text {Pro }}$ as defined in Table.3.

\subsubsection{Intuitions of Main Results}

At first, we discuss the impacts of clustering exponents of friendship degree and friendship formation, i.e., $\gamma$ and $\beta$, on the social-broadcast capacity. We provide an illustration for the protocol model in Fig.5 according to the results of Theorem 4 , and we omit the counterpart for the generalized physical
TABLE 3

Social-Broadcast Capacity under ProM: $\Lambda^{\text {Pro }}$

\begin{tabular}{|c|c|}
\hline$\gamma$ & Social Capacity under ProM $-\Lambda^{\text {Pro }}$ \\
\hline$\gamma>2$ & $\begin{array}{ll}\Theta(1 / \log n), & \beta>2 ; \\
\Theta\left(1 /(\log n)^{\frac{3}{2}}\right), & \beta=2 ; \\
\Theta\left(n^{\frac{\beta}{2}-1} / \sqrt{\log n}\right), & 1<\beta<2 \\
\Theta(1 / \sqrt{n}), & \beta=1 ; \\
\Theta(1 / \sqrt{n \log n}), & 0 \leq \beta<1 .\end{array}$ \\
\hline$\gamma=2$ & $\begin{cases}\Theta\left(1 /(\log n)^{3}\right), & \beta \geq 2 ; \\
\Theta\left(n^{\frac{\beta}{2}-1} / \sqrt{\log n}\right), & 1<\beta<2 ; \\
\Theta(1 / \sqrt{n}), & \beta=1 ; \\
\Theta(1 / \sqrt{n \log n}), & 0 \leq \beta<1 .\end{cases}$ \\
\hline $3 / 2<\gamma<2$ & $\begin{array}{ll}\Theta\left(n^{\gamma-2} / \log n\right), & \beta \geq 2 \gamma-2 ; \\
\Theta\left(n^{\frac{\beta}{2}-1} / \sqrt{\log n}\right), & 1<\beta<2 \gamma-2 ; \\
\Theta(1 / \sqrt{n}), & \beta=1 ; \\
\Theta(1 / \sqrt{n \log n}), & 0 \leq \beta<1 .\end{array}$ \\
\hline$\gamma=3 / 2$ & $\begin{array}{ll}\Theta(1 /(\log n \cdot \sqrt{n})), & \beta \geq 1 ; \\
\Theta(1 /(\log n \cdot \sqrt{n \log n})), & 0 \leq \beta<1 .\end{array}$ \\
\hline $1<\gamma<3 / 2$ & $\Theta\left(1 /\left(\log n \cdot n^{2-\gamma}\right)\right)$ \\
\hline $0 \leq \gamma \leq 1$ & $\Theta(1 / n)$ \\
\hline
\end{tabular}

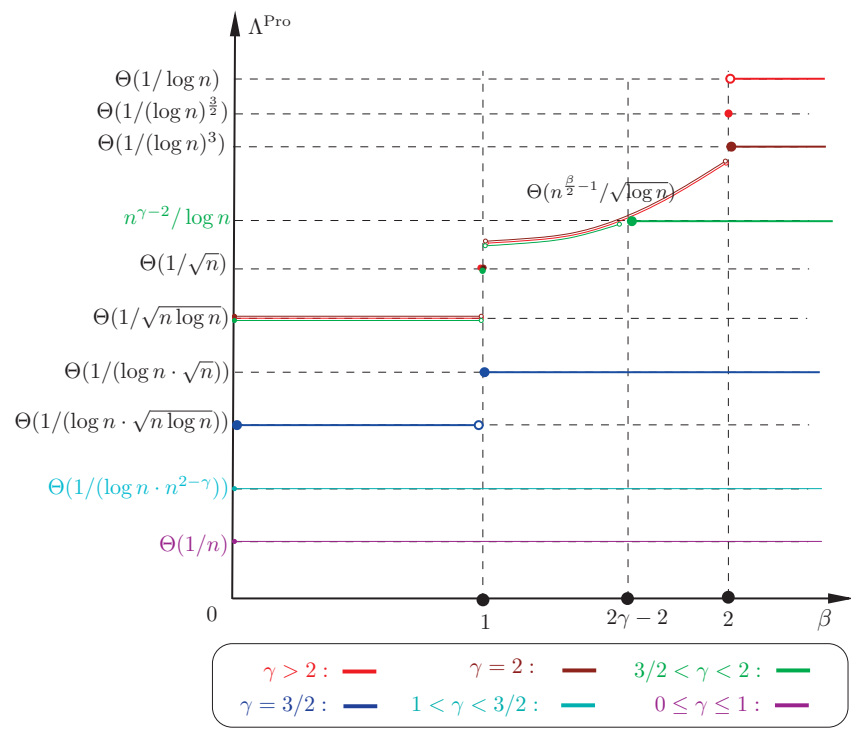

Fig. 5. The Impacts of $\gamma$ and $\beta$ on The Capacity under Protocol Model.

model due to it's similarity of change trend. From Fig.5, the social-broadcast capacity is monotonically nondecreasing in the range $[1 / \log n, 1 / n]$ for both $\gamma$ and $\beta$. An intuitive explanation can be made as follows: A larger clustering exponent of friendship degree $\gamma$ can limit the number of friends of each user into a smaller upper bound with high probability, then leads to a larger social-broadcast capacity (Definition 3); a larger clustering exponent of friendship formation $\beta$ makes the friends more closer to each user with high probability, then possibly reduces the total transmission distance of each social-broadcast session, finally also leads to a larger socialbroadcast capacity.

Importantly, we notice that we only take account of the case that $\delta=0$, where the population-based model degenerates to that similar to distance-based model [17], [31]. The advantages 
of population-based model cannot be sufficiently highlighted for such a special model, indeed. This work can act as the first step of investigating the capacity under the general population-based model. It would be a significant future work to clarify the relationships between the general clustering exponent and capacity in 3-dimensional parameter space, i.e., $(\delta, \gamma, \beta) \in[0, \infty)^{3}$. For such future work, we make a more detailed discussion in Section 5.1 on how to derive the capacity under more general models based on the results in this paper.

\subsubsection{Differences from Existing Work}

As stated above, the distance-based model can be regarded as a special case of the proposed population-based model. In this work, we mainly give the complete results on capacity scaling laws for this special case. Azimdoost et al. [18], [19] had also introduced the distance-based social model into the study of wireless social networks. Naturally, it is necessary to declare the differences between [18], [19] and our results, and highlight the advantages of our work compared with those existing works. We can summarize the differences from three aspects: The first is the different generality of the studied session patterns. Both [18] and [19] focused on the simple unicast capacity by choosing randomly one destination node for each session from the social group (the set of friends) of the source node. They did not give sufficient consideration to the scale-free feature of social relationship distribution and the diversity of session patterns in social applications. While, to address this problem, we investigate the capacity for the social-broadcast sessions where the number of destinations in each session is assumed to follow a Zipf's distribution [26]. The second is the different practicality of the adopted communication models. As two representative communication models, the protocol model and generalized physical model have been widely used in addressing the issue of capacity scaling laws. The former is convenient analytically, while the latter can capture the nature of wireless channels better, then can derive more practical results on network capacity. Both [18] and [19] only investigated the simple protocol model. In our paper, we mainly aim to present more practical results by adopting the generalized physical model, while for completeness, we also include the results under the protocol model. The third is the different scalability of the proposed methods and results. Although only the results on the special case are completely derived in this paper, some proposed analytical methods and results can serve as the basis of addressing network capacity under the general population-based social model with inhomogeneous physical layer. However, the results and methods in [18] and [19] cannot be extended into ones in line with reality for the general physical layer due to the incurable defect of the distance-based model.

\subsection{System Setting}

\subsubsection{Degree Distribution of Social Relationships}

Considering the degree distribution, by Eq.(1), we get that

$$
\operatorname{Pr}\left(q_{k}=l\right)= \begin{cases}\Theta\left(l^{-\gamma}\right), & \gamma>1 \\ \Theta\left(\frac{1}{\log n} \cdot l^{-1}\right), & \gamma=1 \\ \Theta\left(n^{\gamma-1} \cdot l^{-\gamma}\right), & 0 \leq \gamma<1\end{cases}
$$

\subsubsection{Distribution of Anchor Points}

For each session $\mathcal{S}_{k}$ initiated by the source $v_{k}$, we can get the distribution of anchor points directly using Theorem 1,

Lemma 1: When the clustering exponent $\delta=0$, for a session $\mathcal{S}_{k}$ under the population-based social model $\mathbb{P}(\delta=$ $0, \gamma, \beta)$, the anchor points of the friends of source $v_{k}$ follows the distribution of density function:

$f_{v_{k}}(X)= \begin{cases}\Theta\left(\left(\left|X-v_{k}\right|^{2}+1\right)^{-\beta}\right), & \beta>1 ; \\ \Theta\left(\frac{1}{\log n} \cdot\left(\left|X-v_{k}\right|^{2}+1\right)^{-1}\right), & \beta=1 ; \\ \Theta\left(n^{\beta-1} \cdot\left(\left|X-v_{k}\right|^{2}+1\right)^{-\beta}\right), & 0 \leq \beta<1 .\end{cases}$

By using Lemma 1, we can get the following result.

Lemma 2: For a social-broadcast session $\mathcal{S}_{k}$ under the model $\mathbb{P}(\delta=0, \gamma, \beta)$, it holds that:

$$
\mathbf{E}\left[\left|X-v_{k}\right|\right]= \begin{cases}\Theta(1), & \beta>3 / 2 \\ \Theta(\log n), & \beta=3 / 2 ; \\ \Theta\left(n^{\frac{3}{2}-\beta}\right), & 1<\beta<3 / 2 ; \\ \Theta(\sqrt{n} / \log n), & \beta=1 \\ \Theta(\sqrt{n}), & 0 \leq \beta<1 .\end{cases}
$$

\subsubsection{Social-Broadcast Sessions}

Under the population-based social model, we denote a socialbroadcast session by a set $\mathcal{S}_{k}:=\left\{v_{k}\right\} \cup \mathcal{F}_{k}$, where $v_{k}$ is the source node and each element in $\mathcal{F}_{k}=\left\{v_{k_{i}}\right\}_{i=1}^{q_{k}}$, say $v_{k_{i}}$, is the nearest node to the corresponding anchor point $p_{k_{i}}$ in $\mathcal{A}_{k}=\left\{p_{k_{i}}\right\}_{i=1}^{q_{k}}$. Please see the illustration in Fig.2. Recall that $\mathcal{P}_{k}=\left\{v_{k}\right\} \cup \mathcal{A}_{k}$, we get the following Lemma 3 for spanning trees over $\mathcal{S}_{k}$.

Lemma 3: For a social-broadcast session $\mathcal{S}_{k}$ with $q_{k}=$ $\omega(1)$ under the social model $\mathbb{P}(\delta=0, \gamma, \beta)$, with probability 1 , it holds that $\left|\operatorname{EMST}\left(\mathcal{A}_{k}\right)\right|=\Theta\left(L_{\mathcal{P}}\left(\beta, q_{k}\right)\right)$, and then $\left|\operatorname{EMST}\left(\mathcal{P}_{k}\right)\right|=\Omega\left(L_{\mathcal{P}}\left(\beta, q_{k}\right)\right)$, where

$$
L_{\mathcal{P}}\left(\beta, q_{k}\right)= \begin{cases}\Theta\left(\sqrt{q_{k}}\right), & \beta>2 ; \\ \Theta\left(\sqrt{q_{k}} \cdot \log n\right), & \beta=2 ; \\ \Theta\left(\sqrt{q_{k}} \cdot n^{1-\frac{\beta}{2}}\right), & 1<\beta<2 ; \\ \Theta\left(\sqrt{q_{k}} \cdot \sqrt{\frac{n}{\log n}}\right), & \beta=1 ; \\ \Theta\left(\sqrt{q_{k}} \cdot \sqrt{n}\right), & 0 \leq \beta<1 .\end{cases}
$$

Proof: From Theorem 2, it follows that with probability $1,\left|\operatorname{EMST}\left(\mathcal{A}_{k}\right)\right|=\Theta\left(L_{\mathcal{P}}\left(\beta, q_{k}\right)\right)$ for $q_{k}=\omega(1)$, where $L_{\mathcal{P}}\left(\beta, q_{k}\right)$ is defined in Eq.(10). Combining with the fact that $\left|\operatorname{EMST}\left(\mathcal{P}_{k}\right)\right| \geq\left|\operatorname{EMST}\left(\mathcal{A}_{k}\right)\right|$, we get the lemma.

\subsection{Upper Bounds on Social-Broadcast Capacity}

\subsubsection{Technical Preparations for Upper Bounds}

Before computing the upper bounds, we introduce a notion called lattice view.

Definition 4 (Lattice View): Partition a square deployment region $\mathcal{O}(S)=[0, \sqrt{S}]^{2}$ into $\lceil\sqrt{S} / c\rceil^{2}$ cells of side length $c:[\sqrt{S / n}, \sqrt{S})$, we call the produced lattice graph lattice view, and denote it by $\mathbb{V}(\sqrt{S}, c)$.

Based on a given lattice view $\mathbb{V}(\sqrt{S}, c)$, we can get the following lemma for arbitrary routing trees for a session $\mathcal{S}_{k}$. By Lemma 2 of [25], we have 
TABLE 4

$H(\gamma, \beta)$ in Bounding $\sum$ EMST and $\sum$ EST

\begin{tabular}{|c|c|c|}
\hline$\gamma$ & $H(\gamma, \beta)$ & \\
\hline$\gamma>2$ & $\begin{array}{l}\Theta(n) \\
\Theta(n \cdot \log n) \\
\Theta\left(n^{2-\frac{\beta}{2}}\right) \\
\Theta\left(n^{3 / 2} / \sqrt{\log n}\right) \\
\Theta\left(n^{3 / 2}\right)\end{array}$ & $\begin{array}{l}\beta>2 \\
\beta=2 \\
1<\beta<2 \\
\beta=1 \\
0 \leq \beta<1\end{array}$ \\
\hline$\gamma=2$ & $\left\{\begin{array}{l}\Theta(n \cdot \log n) \\
\Theta\left(n^{2-\frac{\beta}{2}}\right) \\
\Theta\left(n^{3 / 2} / \sqrt{\log n}\right) \\
\Theta\left(n^{3 / 2}\right)\end{array}\right.$ & $\begin{array}{l}\beta \geq 2 \\
1<\beta<2 \\
\beta=1 \\
0 \leq \beta<1\end{array}$ \\
\hline $3 / 2<\gamma<2$ & $\left\{\begin{array}{l}\Theta\left(n^{3-\gamma}\right) \\
\Theta\left(n^{2-\frac{\beta}{2}}\right) \\
\Theta\left(n^{3 / 2} / \sqrt{\log n}\right) \\
\Theta\left(n^{3 / 2}\right)\end{array}\right.$ & $\begin{array}{l}\beta \geq 2 \gamma-2 ; \\
1<\beta<2 \gamma-2 ; \\
\beta=1 \\
0 \leq \beta<1\end{array}$ \\
\hline$\gamma=3 / 2$ & $\begin{array}{l}\Theta\left(n^{3 / 2}\right), \\
\Theta\left(n^{3 / 2} \cdot \sqrt{\log n}\right), \\
\Theta\left(n^{3 / 2} \cdot \log n\right),\end{array}$ & $\begin{array}{l}\beta>1 \\
\beta=1 \\
0 \leq \beta<1\end{array}$ \\
\hline $1<\gamma<3 / 2$ & \multicolumn{2}{|l|}{$\Theta\left(n^{3-\gamma}\right)$} \\
\hline$\gamma=1$ & \multicolumn{2}{|l|}{$\Theta\left(n^{2} / \log n\right)$} \\
\hline $0 \leq \gamma<1$ & \multicolumn{2}{|l|}{$\Theta\left(n^{2}\right)$} \\
\hline
\end{tabular}

Lemma 4: Given a social-broadcast session $\mathcal{S}_{k}$, let $\mathcal{T}_{k}$ be a routing tree for $\mathcal{S}_{k}$, and let $N\left(\mathcal{T}_{k}, \sqrt{S}, c\right)$ denote the number of cells used by $\mathcal{T}_{k}$ in $\mathbb{V}(\sqrt{S}, c)$, then when $q_{k}=O\left(S / c^{2}\right)$, it holds that $N\left(\mathcal{T}_{k}, \sqrt{S}, c\right)=\Omega\left(\frac{1}{c} \cdot\left|\operatorname{EMST}\left(\mathcal{S}_{k}\right)\right|\right)$.

In $\mathbb{V}(\sqrt{S}, c)$, a cell is called an island if it contains $\Theta\left(\frac{n c^{2}}{S}\right)$ nodes and all its eight neighbor cells are empty.

Lemma 5 ([25]): There exists w.h.p. an island in the lattice view $\mathbb{V}(\sqrt{S}, c)$, if $c \leq \frac{1}{2} \cdot \sqrt{\frac{(1-\epsilon) \cdot S \cdot \log n}{2 n}}$, where $\epsilon \in(0,1)$ is constant.

Since the sum of length of Euclidean minimum spanning trees for all $n$ sessions, i.e., $\sum_{k=1}^{n}\left|\operatorname{EMST}\left(\mathcal{S}_{k}\right)\right|$, plays a key role in the analysis of network capacity, we need to give the lower bounds on $\sum_{k=1}^{n}\left|\operatorname{EMST}\left(\mathcal{S}_{k}\right)\right|$. Note that the bounds depend on those on $\sum_{k=1}^{n}\left|\operatorname{EMST}\left(\mathcal{P}_{k}\right)\right|$, which will be provided in Lemma B.1.

Lemma 6: For all social-broadcast sessions $\mathcal{S}_{k}, k=$ $1,2, \cdots, n$, under the social model $\mathbb{P}(\delta=0, \gamma, \beta)$, with high probability, $\sum_{k=1}^{n}\left|\operatorname{EMST}\left(\mathcal{S}_{k}\right)\right|=\Omega(H(\gamma, \beta))$, where $H(\gamma, \beta)$ is described in Table.4.

Proof: Please see the proof in Appendix B.1.

\subsubsection{Upper Bounds on Social-Broadcast Capacity}

We will derive the upper bounds by combining the bounds based on the lattice views $\mathbb{V}(\sqrt{n}, \sqrt{2})$ and $\mathbb{V}\left(\sqrt{n}, \frac{1}{3} \sqrt{\log n}\right)$.

Upper Bound from Lattice View $\mathbb{V}(\sqrt{n}, \sqrt{2})$ : Based on Lemma 6, we can get the following lemma.

Lemma 7: Under the social model $\mathbb{P}(\delta=0, \gamma, \beta)$ and the generalized physical model with $\alpha>2$, the per-session socialbroadcast capacity is of order $O(n / H(\gamma, \beta))$, where $H(\gamma, \beta)$ is defined in Table.4.

Proof: Please see the proof in Appendix B.2.
Upper Bound from Lattice View $\mathbb{V}\left(\sqrt{n}, \frac{1}{3} \sqrt{\log n}\right)$ : From Lemma 5, for $\epsilon=\frac{1}{9}$, there is an island in the lattice view $\mathbb{V}\left(\sqrt{n}, \frac{1}{3} \sqrt{\log n}\right)$. Thus, we get the following lemma.

Lemma 8: Under the social model $\mathbb{P}(\delta=0, \gamma, \beta)$ and the generalized physical model with $\alpha>2$, the per-session socialbroadcast capacity is of order

$$
\Lambda= \begin{cases}O\left((\log n)^{-\frac{\alpha}{2}}\right), & \gamma>2 \\ O\left((\log n)^{-\frac{\alpha}{2}-1}\right), & \gamma=2 ; \\ O\left(n^{\gamma-2} \cdot(\log n)^{-\frac{\alpha}{2}}\right), & 1<\gamma<2 \\ O\left((\log n)^{1-\frac{\alpha}{2}} / n\right), & \gamma=1 \\ O\left((\log n)^{-\frac{\alpha}{2}} / n\right), & 0 \leq \gamma<1 .\end{cases}
$$

Proof: Please see the proof in Appendix B.3.

Combination of Upper Bounds: Combining Lemma 7 and Lemma 8, we obtain the upper bounds in Theorem 3 by performing some simple algebraic manipulations.

\subsection{Lower Bounds on Social-Broadcast Capacity}

In this section, we present the constructive lower bounds for the social-broadcast capacity by devising two social-broadcast strategies having their own merits respectively. Both strategies depend on the Euclidean spanning trees (ESTs) of sessions.

\subsubsection{Euclidean Spanning Trees}

Recall that we denote each social-broadcast session by $\mathcal{S}_{k}=$ $\left\{v_{k}\right\} \cup \mathcal{F}_{k}$, where $v_{k}$ is the source node and $\mathcal{F}_{k}$ is the set of friends (or followers) of $v_{k} . \mathcal{A}_{k}=\left\{p_{k_{i}}\right\}_{i=1}^{q_{k}}$ denotes the set of these $q_{k}$ anchor points of the nodes in $\mathcal{F}_{k}=\left\{v_{k_{i}}\right\}_{i=1}^{q_{k}}$.

Construction of Euclidean Spanning Trees: For each session $\mathcal{S}_{k}=\left\{v_{k}\right\} \cup \mathcal{F}_{k}$, we build an $\operatorname{EST}$, denoted as $\operatorname{EST}\left(\mathcal{S}_{k}\right)$, by the following method (Anchors-EMST-Based Greedy (AEBG) Algorithm):

Step 1. Construct a Euclidean minimum spanning tree (EMST) based on $\mathcal{A}_{k}$, denoted as $\operatorname{EMST}\left(\mathcal{A}_{k}\right)$, using some classic greedy algorithms like Prim algorithm.

Step 2. Connect the pairs $v_{k_{i}}$ and $v_{k_{j}}$ if and only if the link $p_{k_{i}} p_{k_{j}} \in \operatorname{EMST}\left(\mathcal{A}_{k}\right)$. Then, one can obtain an EST of $\mathcal{F}_{k}$.

Step 3. Connect the source $v_{k}$ to its nearest node in $\mathcal{F}_{k}$ to get the final EST of $\mathcal{S}_{k}$, i.e., $\operatorname{EST}\left(\mathcal{S}_{k}\right)$.

Bounds on $\sum_{k=1}^{n}\left|\operatorname{EST}\left(\mathcal{S}_{k}\right)\right|$ : We give the upper bounds on $\sum_{k=1}^{n}\left|\operatorname{EST}\left(\mathcal{S}_{k}\right)\right|$.

Lemma 9: For all social-broadcast sessions $\mathcal{S}_{k}, k=$ $1,2, \cdots, n$, under the social model $\mathbb{P}(\delta=0, \gamma, \beta)$, using the aforementioned AEBG algorithm, with high probability, it holds that $\sum_{k=1}^{n}\left|\operatorname{EST}\left(\mathcal{S}_{k}\right)\right|=O(H(\gamma, \beta))$, where $H(\gamma, \beta)$ is described in Table.4.

Proof: Please see the proof in Appendix B.4.

Combining Lemma 6 and Lemma 9, we get that

Theorem 5: For all social-broadcast sessions $\mathcal{S}_{k}$, under the model $\mathbb{P}(\delta=0, \gamma, \beta)$, it holds that with high probability, $\sum_{k=1}^{n}\left|\operatorname{EMST}\left(\mathcal{S}_{k}\right)\right|=\Theta(H(\gamma, \beta))$, where $H(\gamma, \beta)$ is described in Table.4.

\subsubsection{Social-Broadcast Schemes}

Since the physical layer with $\delta=0$ is a random extended network, the percolation-based routing backbone [6] still applies to the social model $\mathbb{P}(\delta=0, \gamma, \beta)$. We introduce two 
TABLE 5

Achievable Throughput under $\mathbb{B}_{\mathrm{p} \& \mathrm{~h}}: \underline{\Lambda}^{\mathbb{B}_{\mathrm{p} \& \mathrm{~h}}}$

\begin{tabular}{|l||ll|}
\hline$\gamma$ & $\Lambda^{\mathbb{B}_{\mathrm{p} \& \mathrm{~h}}}$ \\
\hline \hline \multicolumn{1}{|c||}{} & $\begin{cases}\Omega\left((\log n)^{-\frac{\alpha+1}{2}}\right), & \beta \geq 2 ; \\
\Omega\left(1 / n^{1-\frac{\beta}{2}}\right), & 1<\beta<2 ; \\
\Omega(\sqrt{\log n} / \sqrt{n}), & \beta=1 ; \\
\Omega(1 / \sqrt{n}), & 0 \leq \beta<1 .\end{cases}$ \\
\hline$\gamma=2$ & $\begin{cases}\Omega\left((\log n)^{-\frac{\alpha+3}{2}}\right), & \beta \geq 2 ; \\
\Omega\left(1 / n^{1-\frac{\beta}{2}}\right), & 1<\beta<2 ; \\
\Omega(\sqrt{\log n} / \sqrt{n}), & \beta=1 ; \\
\Omega(1 / \sqrt{n}), & 0 \leq \beta<1 .\end{cases}$ \\
\hline $3 / 2<\gamma<2$ & $\begin{cases}\Omega\left(\frac{(\log n)^{-\frac{\alpha+1}{2}}}{n^{2}-\gamma}\right), & \beta \geq 2 \gamma-2 ; \\
\Omega\left(1 / n^{1-\frac{\beta}{2}}\right), & 1<\beta<2 \gamma-2 ; \\
\Omega(\sqrt{\log n} / \sqrt{n}), & \beta=1 ; \\
\Omega(1 / \sqrt{n}), & 0 \leq \beta<1 .\end{cases}$ \\
\hline $1<\gamma \leq 3 / 2$ & $\Omega\left((\log n)^{-\frac{\alpha+1}{2}} / n^{2-\gamma}\right)$ \\
\hline$\gamma=1$ & $\Omega\left((\log n)^{\frac{1-\alpha}{2}} / n\right)$ \\
\hline $0 \leq \gamma<1$ & $\Omega\left((\log n)^{-\frac{\alpha}{2}} / n\right)$ \\
\hline
\end{tabular}

routing backbones called highways [6] and parallel arterial roads [30] to design the social-broadcast schemes. For the sake of completeness, we include concisely the construction procedures of these backbone systems in Appendix C.1. We propose two types of social-broadcast schemes: The former's hierarchical routing backbone consists of highways and parallel arterial roads, denoted by $\mathbb{B}_{\mathrm{p} \& \mathrm{~h}}$; the latter is only based on parallel arterial roads, denoted by $\mathbb{B}_{\mathrm{p}}$. Due to the relatively insignificant novelty, we move the complete descriptions of these schemes into Appendix C.2.

Next, we give a theorem to present the achievable socialbroadcast throughputs under $\mathbb{B}_{\mathrm{p} \& \mathrm{~h}}$ and $\mathbb{B}_{\mathrm{p}}$.

Theorem 6: Under the social model $\mathbb{P}(\delta=0, \gamma, \beta)$ and generalized physical model with $\alpha>2$, using $\operatorname{EST}\left(\mathcal{S}_{k}\right)$ derived by AEBG algorithm as the input of schemes $\mathbb{B}_{\mathrm{p} \& \mathrm{~h}}$ or $\mathbb{B}_{\mathrm{p}}$, then it holds that with high probability:

$\triangleright$ Under scheme $\mathbb{B}_{\mathrm{p} \& \mathrm{~h}}$, the achievable throughput, denoted by $\underline{\Lambda}^{\mathbb{B}_{p \& h}}$, is described in Table.5.

$\triangleright$ Under scheme $\mathbb{B}_{\mathrm{p}}$, the achievable throughput, denoted by $\underline{\Lambda}^{\mathbb{B}_{\mathrm{p}}}$, is described in Table.6.

Proof: Please see the proof in Appendix C.3.

Combining the throughputs under schemes $\mathbb{B}_{\mathrm{p} \& \mathrm{~h}}$ and $\mathbb{B}_{\mathrm{p}}$ in Theorem 6, we get the lower bounds in Theorem 3 .

\section{Conclusion ANd Future Work}

In this paper, we mainly address capacity scaling laws of wireless social networks under the social-based session formation scheme. A three-layered model is proposed for abstracting wireless social networks. As one of main contributions, a cross-layer and distance\&density-aware social model is proposed, which captures the formation characteristics of realworld social networks better, and specializes in the analysis of capacity scaling laws. We derive the social-broadcast capacity, taking into account the general clustering exponents of both friendship degree and friendship formation. Moreover, we
TABLE 6

Achievable Throughput under $\mathbb{B}_{\mathrm{p}}: \underline{\Lambda}^{\mathbb{B}_{\mathrm{p}}}$

\begin{tabular}{|c|c|c|}
\hline$\gamma$ & $\underline{\Lambda}^{\mathbb{B}_{\mathrm{p}}}$ & \\
\hline$\gamma>2$ & $\begin{array}{l}\Omega\left((\log n)^{-\frac{\alpha}{2}}\right), \\
\Omega\left((\log n)^{-\frac{\alpha+1}{2}}\right), \\
\Omega\left((\log n)^{\frac{1-\alpha}{2}} / n^{1-\frac{\beta}{2}}\right), \\
\Omega\left((\log n)^{\frac{2-\alpha}{2}} / \sqrt{n}\right), \\
\Omega\left((\log n)^{\frac{1-\alpha}{2}} / \sqrt{n}\right),\end{array}$ & $\begin{array}{l}\beta>2 \\
\beta=2 \\
1<\beta<2 \\
\beta=1 \\
0 \leq \beta<1 .\end{array}$ \\
\hline$\gamma=2$ & $\begin{array}{l}\Omega\left((\log n)^{-\frac{\alpha}{2}-2}\right), \\
\Omega\left((\log n)^{\frac{1-\alpha}{2}} / n^{1-\frac{\beta}{2}}\right), \\
\Omega\left((\log n)^{\frac{2-\alpha}{2}} / \sqrt{n}\right) \\
\Omega\left((\log n)^{\frac{1-\alpha}{2}} / \sqrt{n}\right)\end{array}$ & $\begin{array}{l}\beta \geq 2 \\
1<\beta<2 \\
\beta=1 \\
0 \leq \beta<1\end{array}$ \\
\hline $3 / 2<\gamma<2$ & $\begin{array}{l}\Omega\left((\log n)^{-\frac{\alpha}{2}} / n^{2-\gamma}\right), \\
\Omega\left((\log n)^{\frac{1-\alpha}{2}} / n^{1-\frac{\beta}{2}}\right), \\
\Omega\left((\log n)^{\frac{2-\alpha}{2}} / \sqrt{n}\right) \\
\Omega\left((\log n)^{\frac{1-\alpha}{2}} / \sqrt{n}\right)\end{array}$ & $\begin{array}{l}\beta \geq 2 \gamma-2 ; \\
1<\beta<2 \gamma-2 ; \\
\beta=1 \\
0 \leq \beta<1\end{array}$ \\
\hline$\gamma=3 / 2$ & $\begin{array}{l}\Omega\left((\log n)^{-\frac{\alpha}{2}} / \sqrt{n}\right) \\
\Omega\left((\log n)^{-\frac{\alpha+1}{2}} / \sqrt{n}\right)\end{array}$ & $\begin{array}{l}\beta \geq 1 \\
0 \leq \beta<1\end{array}$ \\
\hline $1 \leq \gamma<3 / 2$ & \multicolumn{2}{|l|}{$\Omega\left((\log n)^{-\frac{\alpha}{2}} / n^{2-\gamma}\right)$} \\
\hline $0 \leq \gamma<1$ & \multicolumn{2}{|l|}{$\Omega\left((\log n)^{-\frac{\alpha}{2}} / n\right)$} \\
\hline
\end{tabular}

present the density function of general social friendships distribution that will be the basis for investigating the capacity of general wireless social networks. This work can act as the first step of investigating the capacity under the proposed population-based model.

Next, we make a discussion on how to derive the capacity under more general models based on the results of this work.

\subsection{Extending to General Physical Clustering Case}

The advantages of population-based model cannot be sufficiently highlighted for the case that $\delta=0$, indeed. It would be a significant future work to investigate the relationships between the general clustering exponent and capacity in 3dimensional parameter space, i.e., $(\delta, \gamma, \beta) \in[0, \infty)^{3}$.

Here, we probe the feasibility of studying network capacity under the social model with inhomogeneous physical layer, by extending the proposed basic theorem (Theorem 1) for the general distribution of anchor points. The key factor is to determine $\mathbf{d}(Y)$. Furthermore, since $\mathbf{d}(Y)=\frac{n \cdot \min \left\{1,|Y-O|^{-\delta}\right\}}{\int_{\mathcal{O}} \min \left\{1,|Z-O|^{-\delta}\right\} d Z}$, then it should be the first key step to deal with $|Y-O|$ based on the "known" distance deviating from the source $L_{s}=\left|Y-v_{k}\right|$ and distance deviating from the center $L_{c}=\left|v_{k}-O\right|$. As illustrated in Fig.6, it follows that

$$
|Y-O|=\sqrt{L_{c}^{2}+L_{s}^{2}-2 L_{c} \cdot L_{s} \cdot \cos \theta},
$$

which can serve as a basis for the further study on the general model.

\subsection{Extending to General Physical Layer}

The proposed center-clustering random model (CCRM) can be developed to more general physical layer models in terms of clustering patterns and scaling patterns. 


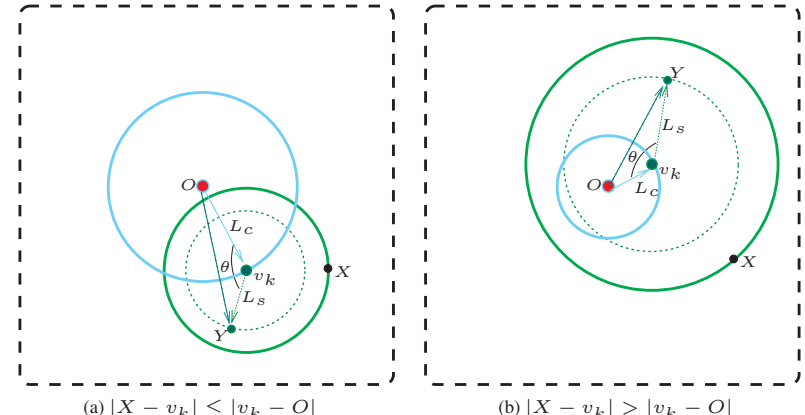

Fig. 6. Illustrations of positions of the center point $O$, the reference point $v_{k}$, the targeted point $X$, and any interior point $Y$ in $\mathcal{D}\left(v_{k},\left|X-v_{k}\right|\right)$.

\subsubsection{Multiple Clustering Centers}

In the CCRM, there is only one center. Although the results under this model also apply to the model with physical layer model having a finite number of centers due to the characteristic of capacity scaling issues, more realistic and general clustering behaviors in real-life networks cannot be fully embodied by this simple model. To model the clustering behavior of node distribution with $\omega(1)$ centers in real-life applications of wireless networks, the shotnoise Cox process (SNCP, [23], [32]) can be introduced, where $M$ center points generate their respective point processes, and the conditional local intensity at $X$ is determined by the superposition of the individual processes, i.e., $\mathbf{d}(X)=\sum_{j} \rho_{j} \cdot \kappa\left(c_{j}, X\right)$, Then, the node process forms an inhomogeneous Poisson point process.

\subsubsection{General Scaling Models}

In the research of network capacity scaling laws, there are two typical models in terms of scaling patterns of network: dense scaling model and extended scaling model [6], [30], [33]. They have different engineering implications related to the classical notions of interference-limitedness and coverage-limitedness [33]: The former is only interference-limited; while, the latter is both interference-limited and coverage-limited. In the CCRM adopted in this paper, the area of deployment region is $S:=n$, which implies that it is of extended scaling pattern. Then, it is necessary to extend the CCRM to one with general scaling pattern by setting a general area of deployment region. Such a work will directly involve defining the dispersion density function $g(\cdot)$ and power attenuation function $\ell(\cdot)$ which might have a significant impact on the network capacity. Unlike the setting for extended scaling models, these two functions for dense scaling models are usually defined as: $g(s):=s^{-\delta}$ [23] and $\ell(s):=s^{-\alpha}[6]$, respectively.

\subsection{Extending to General Underlying Networks}

For addressing the performance of data transmission in underlying communication networks for social networking services (SNSs), besides the architecture of specific SNSs, there is another key factor to consider. It is the architecture of underlying communication network, e.g., wireless, wired, or hybrid networking architectures. This has a significant impact on implementing routing for specific data dissemination sessions, and plays a key role in limiting network capacity. In this paper, we focus on the scenario where social networking applications operate on the underlying wireless ad hoc networks, and aim to investigate the capacity scaling laws of a large-scale ad hoc network when it undertakes the data transmission of social applications. However, we notice that the real-world underlying network for social networking applications is most unlikely a pure wireless ad hoc networks. A realistic and effective underlying network might be a hybrid network consisting of the Internet and different types of wireless networks, including static wireless and mobile networks. Therefore, it will be a very challenging but significant future work to extend the study by considering the diversity of real-world underlying networks, based on the preliminary results on wireless ad hoc networks in this work. For example, the next feasible and necessary extension could be addressing the fundamental limits of mobile social ad hoc networks where the underlying communication networks can be modeled as mobile ad hoc networks.

\section{ACKNOWLEDGEMENT}

The research of authors is partially supported by the National Natural Science Foundation of China (NSFC) under grant No. 61202383, Shanghai Rising-Star Program under grant No. 14QA1403700, the Program for New Century Excellent Talents in University (NCET) under grant No. NCET-120414, the Natural Science Foundation of Shanghai under grant No. 12ZR1451200, the Integrated Project for Major Research Plan of the National Natural Science Foundation of China under grant No. 91218301, and the Research Fund for the Doctoral Program of Higher Education of China (RFDP) under grant No. 20120072120075. The research of XiangYang Li is partially supported by NSF CNS-0832120, NSF CNS-1035894, NSF ECCS-1247944, NSF ECCS-1343306, National Natural Science Foundation of China under Grant No. 61170216, No. 61228202. Any opinions, findings, conclusions, or recommendations expressed in this paper are those of author(s) and do not necessarily reflect the views of the funding agencies (NSF, and NSFC).

\section{REFERENCES}

[1] Y. Ahn, S. Han, H. Kwak, S. Moon, and H. Jeong, "Analysis of topological characteristics of huge online social networking services," in Proc. ACM WWW 2007.

[2] H. Kwak, C. Lee, H. Park, and S. Moon, "What is twitter, a social network or a news media?" in Proc. ACM WWW 2010.

[3] A. Mislove, H. Koppula, K. Gummadi, P. Druschel, and B. Bhattacharjee, "Growth of the flickr social network," in Proc. ACM Workshop on Online social networks 2008.

[4] A. Mislove, M. Marcon, K. Gummadi, P. Druschel, and B. Bhattacharjee, "Measurement and analysis of online social networks," in Proc. ACM IMC 2007.

[5] B. Viswanath, A. Mislove, M. Cha, and K. Gummadi, "On the evolution of user interaction in facebook," in Proc. ACM Workshop on Online Social Networks 2009.

[6] M. Franceschetti, O. Dousse, D. Tse, and P. Thiran, "Closing the gap in the capacity of wireless networks via percolation theory," IEEE Transactions on Information Theory, vol. 53, no. 3, pp. 1009-1018, 2007.

[7] Q. Peng, X. Wang, and H. Tang, "Heterogeneity increases multicast capacity in clustered network," in Proc. IEEE INFOCOM 2011. 
[8] L. Ying, S. Yang, and R. Srikant, "Optimal delay-throughput tradeoffs in mobile ad hoc networks," IEEE Transactions on Information Theory, vol. 54, no. 9, pp. 4119-4143, 2008.

[9] P. Li, C. Zhang, and M. Y. Fang, "Capacity and delay of hybrid wireless broadband access networks," IEEE Journal on Selected Areas in Communications, vol. 27, no. 2, pp. 117-125, 2009.

[10] — "The capacity of wireless ad hoc networks using directional antennas," IEEE Transactions on Mobile Computing, vol. 10, no. 10, pp. 1374-1387, 2011.

[11] X. Wang, L. Fu, and C. Hu, "Multicast performance with hierarchical cooperation," IEEE/ACM Transactions on Networking, vol. 20, no. 3, pp. 917-930, 2012.

[12] N. Lu, T. H. Luan, M. Wang, X. Shen, and F. Bai, "Capacity and delay analysis for social-proximity urban vehicular networks," in Proc. IEEE INFOCOM 2012.

[13] P. Gupta and P. R. Kumar, "The capacity of wireless networks," IEEE Transactions on Information Theory, vol. 46, no. 2, pp. 388-404, 2000.

[14] S. Milgram, "The small world problem," Psychology today, vol. 2, no. 1, pp. 60-67, 1967.

[15] P. Killworth and H. Bernard, "The reversal small-world experiment," Social networks, vol. 1, no. 2, pp. 159-192, 1979.

[16] P. Dodds, R. Muhamad, and D. Watts, "An experimental study of search in global social networks," science, vol. 301, no. 5634, pp. 827-829, 2003.

[17] J. Kleinberg, "Navigation in a small world," Nature, vol. 406, no. 6798, pp. $845-845,2000$.

[18] B. Azimdoost, H. Sadjadpour, and J. Garcia-Luna-Aceves, "Capacity of composite networks: Combining social and wireless ad hoc networks," in Proc. IEEE WCNC 2011.

[19] B. Azimdoost and H. Sadjadpour, "Capacity of scale free wireless networks," in Proc. IEEE GlobeCom 2012.

[20] D. Liben-Nowell, J. Novak, R. Kumar, P. Raghavan, and A. Tomkins, "Geographic routing in social networks," PNAS of the United States of America, vol. 102, no. 33, pp. 11 623-11 628, 2005.

[21] S. Li, Y. Liu, and X.-Y. Li, "Capacity of large scale wireless networks under Gaussian channel model," in Proc. ACM MobiCom 2008.

[22] L. Xiang-Yang., S. Tang, and F. Ophir, "Multicast capacity for large scale wireless ad hoc networks," in Proc. ACM MobiCom 2007.

[23] G. Alfano, M. Garetto, and E. Leonardi, "Capacity scaling of wireless networks with inhomogeneous node density: Upper bounds," IEEE Journal on Selected Areas in Communications, vol. 27, no. 7, pp. 11471157, 2009

[24] G. Alfano, M. Garetto, E. Leonardi, and V. Martina, "Capacity scaling of wireless networks with inhomogeneous node density: Lower bounds," IEEE/ACM Transactions on Networking, vol. 18, no. 5, pp. 1624-1636, 2010.

[25] C. Wang, C. Jiang, X.-Y. Li, S. Tang, Y. He, X. Mao, and Y. Liu, "Scaling laws of multicast capacity for power-constrained wireless networks under gaussian channel model," IEEE Transactions on Computers, vol. 61 , no. 5, pp. 713-725, 2012.

[26] C. Manning and H. Schütze, Foundations of statistical natural language processing. MIT press, 1999.

[27] V. Lenders, M. May, and B. Plattner, "Density-based anycast: a robust routing strategy for wireless ad hoc networks," IEEE/ACM Transactions on Networking, vol. 16, no. 4, pp. 852-863, 2008.

[28] C. Carter, S. Yi, P. Ratanchandani, and R. Kravets, "Manycast: exploring the space between anycast and multicast in ad hoc networks," in Proc. ACM MobiCom 2003.

[29] M. Penrose, "A strong law for the longest edge of the minimal spanning tree," The Annals of Probability, vol. 27, no. 1, pp. 246-260, 1999.

[30] C. Wang, C. Jiang, X.-Y. Li, S. Tang, and P. Yang, "General capacity scaling of wireless networks," in Proc. IEEE INFOCOM 2011.

[31] B. Latané, J. Liu, A. Nowak, M. Bonevento, and L. Zheng, "Distance matters: Physical space and social impact," Personality and Social Psychology Bulletin, vol. 21, no. 8, pp. 795-805, 1995.

[32] J. Møller, "Shot noise cox processes," Advances in Applied Probability, vol. 35, no. 3, pp. 614-640, 2003.

[33] A. ÖzgÜr, O. LÉvÊque, and D. Tse, "Hierarchical Cooperation Achieves Optimal Capacity Scaling in Ad Hoc Networks," IEEE Transactions on Information Theory, vol. 53, no. 10, pp. 3549-3572, 2007.

[34] J. Steele, "Growth rates of euclidean minimal spanning trees with power weighted edges," The Annals of Probability, pp. 1767-1787, 1988.

[35] D. Williams, Probability with martingales. Cambridge university press, 1991.

[36] N. Z. Gong, W. Xu, and D. Song, "Reciprocity in social networks: Measurements, predictions, and implications," arXiv preprint arXiv:1302.6309, 2013
[37] N. Z. Gong, T. Ameet, M. Lester, H. Ling, S. Eui Chul Richard, S. Emil, S. Elaine(Runting), and S. Dawn, "Joint link prediction and attribute inference using a social-attribute network," Accepted by ACM Transaction on Intelligent Systems and Technology, 2013.

[38] N. Z. Gong, W. Xu, L. Huang, P. Mittal, E. Stefanov, V. Sekar, and D. Song, "Evolution of social-attribute networks: measurements, modeling, and implications using google+," in Proc. ACM IMC 2012.

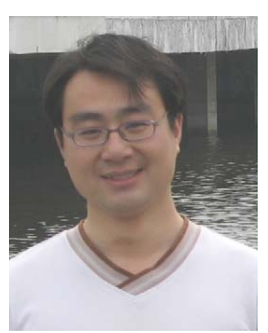

Cheng Wang received his $\mathrm{PhD}$ degree in Department of Computer Science at Tongji University in 2011. Currently, he is a research professor of Computer Science at Tongji University. His research interests include wireless networking, mobile social networks, and cloud computing.

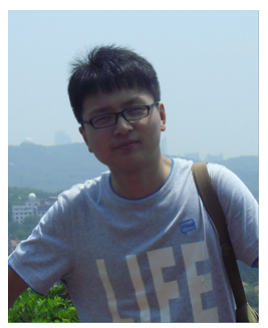

Lu Shao received his bachelor degree of science at College of Science from China University of Petroleum in June, 2013. He is now a master student at Department of Computer Science at Tongji University in Shanghai, China. His research interests include wireless networks and distributed computing.

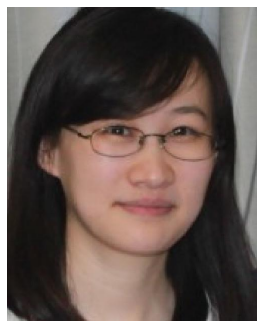

Zhong Li received her B.S. and M.S. degree at Department of Computer Science and Technology from Shandong Normal University in 2007 and 2010 , respectively. She is currently a Ph.D. student in Department of Computer Science at Tongji University in Shanghai, China. Her research interests include wireless communication, social network analysis and distributed computing.

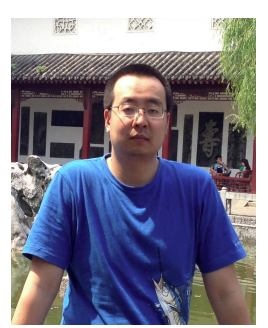

Lei Yang received the BS degree in the School of Software and PhD degree in Computer Science and Technology from Xian Jiaotong University, China, in 2004 and 2014, respectively. He is currently with the School of Software in Tsinghua University. His research interests include RFID, ambient backscatter, pervasive computing, smart home, and communication security. He is a member of the IEEE and the IEEE computer society.

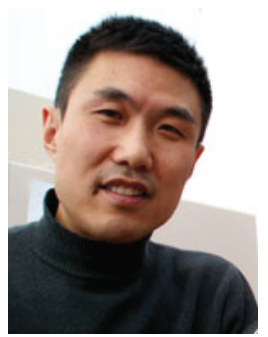

Xiang-Yang Li (M'99, SM'08) is a professor at the Illinois Institute of Technology. He holds EMC -Endowed Visiting Chair Professorship at Tsinghua University. He is a recipient of China NSF Outstanding Overseas Young Researcher (B). Dr. Li received MS (2000) and PhD (2001) degree at Department of Computer Science from University of Illinois at Urbana-Champaign, a Bachelor degree at Department of Computer Science and a Bachelor degree at Department of Business Management from Tsinghua University, P.R. China, both in 1995. His research interests include mobile computing, cyber physical systems, wireless networks, security and privacy, and algorithms. He published a monograph "Wireless Ad Hoc and Sensor Networks: Theory and Applications". He co-edited several books, including, "Encyclopedia of Algorithms". Dr. Li is an editor of several journals, including IEEE Transaction on Parallel and Distributed Systems, IEEE Transaction on Mobile Computing. He has served many international conferences in various capacities, including ACM MobiCom, ACM MobiHoc, ACM STOC, IEEE MASS. He is a senior member of IEEE and a member of ACM. The research of Xiang-Yang Li is partially supported by USA NSF, National Natural Science Foundation of China, and RGC of HongKong. 


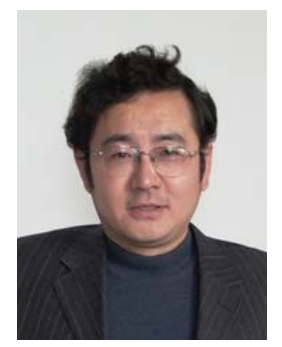

Changjun Jiang received the Ph.D. degree from the Institute of Automation, Chinese Academy of Sciences, Beijing, China, in 1995. Currently he is a Professor with the Department of Computer Science and Engineering, Tongji University, Shanghai. His current areas of research are con- current theory, Petri net, and formal verification of software, wireless networks, concurrency processing and intelligent transportation systems. 